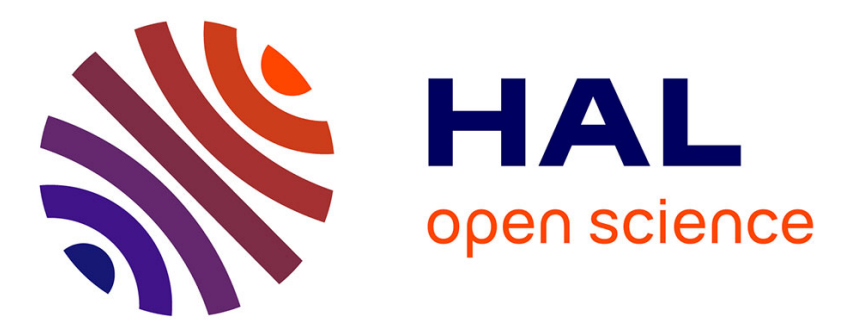

\title{
DIFFUSION DE L'ALUMINIUM DANS LE SILICIUM CRISTALLIN PAR RECUIT LASER SEMI-CONTINU
}

\author{
C. Leray, J. Bouree, M. Rodot
}

\section{To cite this version:}

C. Leray, J. Bouree, M. Rodot. DIFFUSION DE L'ALUMINIUM DANS LE SILICIUM CRISTALLIN PAR RECUIT LASER SEMI-CONTINU. Journal de Physique Colloques, 1983, 44 (C5), pp.C5-235-

C5-240. 10.1051/jphyscol:1983537 . jpa-00223123

\section{HAL Id: jpa-00223123 https://hal.science/jpa-00223123}

Submitted on 1 Jan 1983

HAL is a multi-disciplinary open access archive for the deposit and dissemination of scientific research documents, whether they are published or not. The documents may come from teaching and research institutions in France or abroad, or from public or private research centers.
L'archive ouverte pluridisciplinaire HAL, est destinée au dépôt et à la diffusion de documents scientifiques de niveau recherche, publiés ou non, émanant des établissements d'enseignement et de recherche français ou étrangers, des laboratoires publics ou privés. 


\title{
DIFFUSION DE L'ALUMINIUM DANS LE SILICIUM CRISTALLIN PAR RECUIT LASER SEMI-CONTINU
}

\author{
C. Leray, J.E. Bouree et M. Rodot \\ Laboratoire de Physique des Solides, CNRS, 1 pl. A. Briand, \\ 92190 Meudon, Erance
}

\begin{abstract}
Résumé - La diffusion atomique de l'aluminium dans le silicium monocristallin et polycristallin à petits grains (diamètre voisin de $10 \mu \mathrm{m}$ ) a été étudiée dans le domaine de température $1100-1400^{\circ} \mathrm{C}$. Les échantillons subissent une implantation d'aluminium, un recuit laser semi-continu en phase solide, puis une analyse au microanalyseur ionique. Utilisant un modèle thermique dérivé de celui de GOLD et GIBBONS, on retrouve pour les monocristaux entre $1400^{\circ} \mathrm{C}$ et $1200^{\circ} \mathrm{C}$ le coefficient de diffusion $D_{V}$ généralement admis, d'énergie d'activation $3,4 \mathrm{eV}$; dans les polycristaux apparât au-dessous de $1300^{\circ} \mathrm{C}$ une diffusion intergranulaire $D_{j}$, d'énergie d'activation beaucoup plus faible.

Abstract - The atomic diffusion of aluminum in single crystal or fine-grain polycristalline $\mathrm{Si}$ has been studied in the temperature range $1100-1400^{\circ} \mathrm{C}$. The samples are A1-implanted, then annealed (in so1id phase) by semi-continuous laser annealing and finally analysed using an ion-analyser. Using a thermal model derived from that of GOLD and GIBBONS, we find for single crystals, in the range $1200^{\circ} \mathrm{C}-1400^{\circ} \mathrm{C}$, the generally admitted diffusion coefficient $\mathrm{D}_{\mathrm{v}}$, with an activation energy $3.4 \mathrm{eV}$; in polycrystals below $1300^{\circ} \mathrm{C}$ appears the contribution of intergranular diffusion $\mathrm{D}_{j}$, with a much lower activation energy.
\end{abstract}

\section{INTRODUCTION}

Le recuit laser en phase solide introduit une nouvelle méthode efficace pour 1 'étude de la diffusion atomique dans les semiconducteurs /1/. Cette technique de diffusion, similaire au recuit thermique, permet : une localisation spatiale de la diffusion $(\phi 100 \mu \mathrm{m})$, une montée brutale de température $\left(1400^{\circ} \mathrm{C}\right.$ en quelques ms pour Si) et un temps de recuit court $(<1 \mathrm{s.})$. Elle est donc particulièrement bien ađaptée à une diffusion rapide comme celle du fer (instertitiel) dans le silicium $\left(D=10^{-5} \mathrm{~cm}^{2} / \mathrm{s}\right.$ vers $\left.1400^{\circ} \mathrm{C}\right) / 2,3 /$, où une durée de recuit de $15 \mathrm{~ms}$ induit une longueur de diffusion de plusieurs milliers d'angströms mesurable à l'aide d'un microanalyseur ionique.

Par contre l'aluminium, comme la plupart des éléments des groupes III et V, se comporte dans le silicium en impureté substitutionnelle et de ce fait diffuse relativement lentement (o $2 \cdot 10^{-10} \mathrm{~cm}^{2} / \mathrm{s}$ à $1400^{\circ} \mathrm{C}$ ) $/ 4,5 /$. Nous montrons que notre méthode peut également s'appliquer à l'étude d'une impureté diffusant aussi lentement que 1 'aluminium. Nous avons voulu comparer 1 a diffusion de Al dans Si monocristallin et polycristallin; en effet la diffusion intergranulaire semble n'avoir été mesurée qu ${ }^{\prime}$ entre 350 et $425^{\circ} \mathrm{C} / 6 /$.

Une application de cette étude concerne la croissance de couches CVD pour les photopiles solaires épitaxiées. La connaissance de $D(T)$ commande celle de la teneur tolérable d'impuretés dans le substrat, et il est important de savoir si cette limite est abaissée dans le cas du polycristal à cause de la diffusion intergranulaire.

Ce travail présente les résultats de mesure du coefficient D de Al dans Si obtenus à différentes températures à partir du recuit laser semi-continu : laser continu 
associé à un hacheur mécanique de façon à créer des impulsions longues (quelques fractions de seconde). D se déduit de l'analyse des profils de concentration mesurés au microanalyseur ionique, comparée au modèle théorique /3/ que nous avons développé à partir des idées de GOLD et GIBBONS $/ 7 /$.

\section{MODELE THERMIQUE DU RECUIT LASER}

Notre analyse théorique de la diffusion induite par recuit laser en phase solide comprend deux parties distinctes :

- modélisation de la distribution de température au sein de l'échantillon en fonction du temps.

- modélisation de la diffusion induite par cette distribution de température.

La première partie a fait 1 'objet de nombreux travaux ( $\operatorname{LAX} / 8 /$, NISSIM $19 /$, etc...) dont nous nous sommes inspirés. Comme nous le détaillerons dans une publication ultérieure, notre modèle est validé par des mesures indépendantes du rayon gaussien $\omega$ de la tache laser et de la puissance laser $\mathrm{P}_{\mathrm{f}}$ nécessaire pour la fusion, en fonction du temps d'impulsion $\tau$.

Dans la seconde partie nous utilisons le fait que la diffusion thermique suit une loi d'Arrhénius. Notre modèle montre alors que la diffusion observée lors d'un recuit laser peut être assimilée à un recuit thermique à une température $\mathrm{T}_{\text {eff }}$ égale à la température maximale atteinte lors du recuit et pendant un temps $t$ eff fonction des divers paramètres du recuit $(\tau, P, \omega)$ et de 1 'énergie d'activation $E_{a}$ de 1 'espèce diffusante.

\section{CONDITIONS EXPERIMENTALES}

a - Préparation des échantín lilons

Les échantilions proviennent de Wacker-Heliotronic et sont :

- soit monocristallins, d'orientation (111) et de faible dopage en bore $(1 \Omega . \mathrm{cm})$, - soit polycristallins à petits grains (grain allongé de largeur moyenne $3 \mu \mathrm{m}$ ) et faiblement dopés en $B(1 \Omega . \mathrm{cm})$.

Après un polissage mécanique, tous les échantillons sont dégraissés et attaqués chimiquement avec le réactif $10 \mathrm{HNO}_{3}+4 \mathrm{CH}_{3} \mathrm{COOH}+1 \mathrm{HF}$ pendant 3 minutes.

Juste avant I'introduction dans la chambre d'implantation, dans I'enceinte de recuit ou dans le microanalyseur, les échantillons sont attaqués $1 \mathrm{mn}$ dans $\mathrm{HF}$ (20\%) et lavés à l'eau distillée.

b - Implantation ionionique ( $\mathrm{J}$. CHAUMONT, Laboratoire René Bernas, Orsay)

Les ions $\mathrm{Al}^{+}$sont implantés àdes doses de $10^{14} \mathrm{~cm}^{-2}$ ou $10^{15} \mathrm{~cm}^{-2}$, avec des énergies égales à $30 \mathrm{keV}$ ou $100 \mathrm{keV}$, de façon telle que les concentrations maximales d'impuretés soient respectivement inférieures et supérieures à la limite de solubilité.

\section{c - Recuit laser semi-continu}

Le dispositif de recuit mis au point par R. LEGROS et J. PLASSARD comprend un laser $\mathrm{Ar}^{+}$continu de puissance maximale 20 watts émettant dans le domaine 488-514 nm, un disque rotatif obturant le faisceau laser à intervalle régulier, un système de relais couplé à un microcalculateur ainsi que des lentilles de focalisation. Les durées d'impulsion $\tau$ peuvent varier entre $10 \mu \mathrm{s}$ et $100 \mathrm{~ms}$. Entre deux impulsions successives le microcalculateur commande le déplacement de la platine sur laquelle est fixé 1'échantilion, suivant un pas prédéterminé dans les deux directions perpendiculaires à l'axe du faisceau laser. On peut ainsi faire un recuit homogène sur une surface carrée de côté $1 \mathrm{~mm}$ et réaliser différentes conditions de recuit sur un même échantillon de surface $1 \mathrm{~cm}^{2}$.

Enfin 1'enceinte de recuit comprend un circuit d'injection de gaz et une chaufferette couplée à un système d'asservissement de la température. Par ce système $1 \mathrm{a}$ face arrière de $1^{\prime}$ échantillon est régulée à $2^{\circ} \mathrm{C}$ près entre la température ambiante 
et $300^{\circ} \mathrm{C}$, quelles que soient 1a puissance $P$ du faisceau laser et la durée d'impulsion $\tau$. Pendant le recuit l'échantilion se trouve sous atmosphère contrôlée (hélium ou argon pour éviter la diffusion d'oxygène).

En conséquence cinq paramètres expérimentaux sont contrôlés durant le recuit : la puissance $P$ du faisceau, la durée d'impulsion $\tau$, la rayon gaussien $\omega$ de la tache laser, la distance $\mathrm{d}$ entre deux taches consécutives et la température du substrat $\mathrm{T}_{\mathrm{a}}$.

d - Méthode d'analyse

Un mícroanalyseur ionique Cameca type SMI 300 est utilisé en mode image (diamètre de de la sonde $70 \mu \mathrm{m}$ ) avec un balayage carré de côté 300 à $400 \mu \mathrm{m})$. Les ions primaires $0_{2}^{+}$sont accélérés à $5,5 \mathrm{kV}$ et $1 \mathrm{e}$ courant associé est voisin de $20 \mathrm{nA}$. Les ions secondaires analysés sont ${ }^{27} \mathrm{Al}^{+},{ }^{28} \mathrm{Si}^{+},{ }^{29} \mathrm{Si}^{+}$. Les profils en intégration alternée sont relevés sur une profondeur de l'ordre de $0,4 \mu \mathrm{m}$, avec une vitesse d'érosion voisine de $100 \AA / \mathrm{mn}$; ces valeurs sont déduites de mesures au Talystep. Le rapport des intensités de raie ${ }^{27} \mathrm{Al}^{+}$et ${ }^{28} \mathrm{Si}^{+}$est choisi pour représenter la concentration d'aluminium.

\section{RESULTATS ET DISCUSSION}

Pour les conditions fixées de recuit : $\omega=34 \mu \mathrm{m}, \tau=100 \mathrm{~ms}$, réseau hexagonal de pas $10 \mu \mathrm{m}, \mathrm{T}_{\mathrm{a}}=19^{\circ} \mathrm{C}$ ou $300^{\circ} \mathrm{C}$, les résultats relatifs aux monocristaux implantés $10^{15} \mathrm{~cm}^{-2}$ ( $30 \mathrm{keV}$ ou $100 \mathrm{keV}$ ) et aux polycristaux implantés $10^{14} \mathrm{~cm}^{-2}$ (30 keV) sont présentés dans les Fig. 1, 2 et 3 . Les profils d'Al sont normalisés en égalant à 1. la concentration maximale du profil avant recuit.

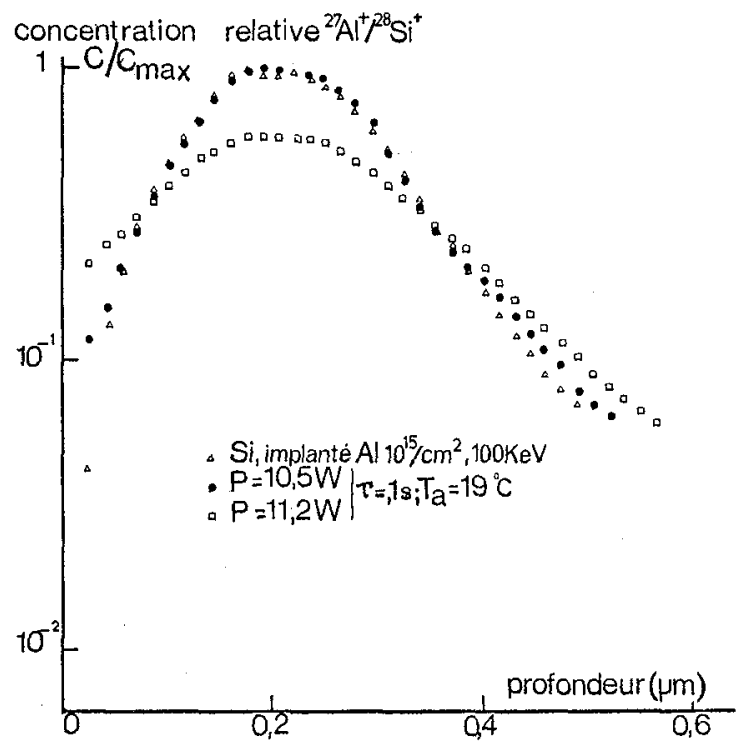

Fig. 1 - Profils de concentration d'aluminium d'un échantillon monocristallin implanté $\mathrm{Al}=10^{15} \mathrm{~cm}^{-2}$, $100 \mathrm{keV}$, et du même échantillon après recuit 1aser. La température du substrat $\mathrm{T}_{\mathrm{a}}$ est maintenue à $19^{\circ} \mathrm{C}$.

Aluminum concentration profiles obtained for as-implanted $\left(10^{15} \mathrm{~cm}^{-2}\right.$, $100 \mathrm{keV}$ ) and laser annealed single crystal $\mathrm{Si}$ sample. Regulated substrate temperature is $\mathrm{T}_{\mathrm{a}}=19^{\circ} \mathrm{C}$. 


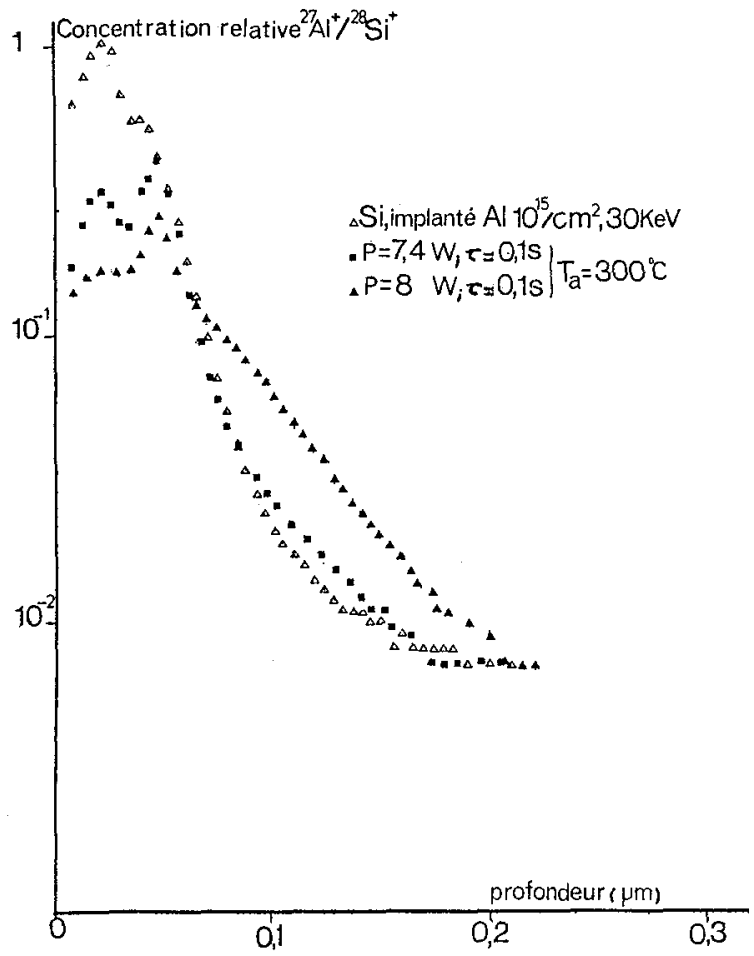

Fig. 2 - Profils de concentration d'aluminium d'un échantillon monocristallin implanté $A l=10^{15} \mathrm{~cm}^{-2}, 30 \mathrm{keV}$, et du même échantillon après recuit laser. La température du substrat $T_{a}$ est maintenue à $300^{\circ} \mathrm{C}$.

Aluminium concentration profiles obtained for as-implanted $\left(10^{15} \mathrm{~cm}^{-2}, 30 \mathrm{keV}\right)$ and laser annealed single crystal Si sample. Regulated substrate temperature is $\mathrm{T}_{\mathrm{a}}=300^{\circ} \mathrm{C}$.

Fig. 3 - Profils de concentration d'aluminium d'un échantillon polycristallin implanté $\mathrm{A} 1=10^{14} \mathrm{~cm}^{-2}, 30 \mathrm{keV}$, et du même échantilion après recuit laser. La température $\mathrm{du}$ substrat $\mathrm{T}_{\mathrm{a}}$ est maintenue à $19^{\circ} \mathrm{C}$.

Aluminium concentration profiles obtained for as-implanted $\left(10^{14} \mathrm{~cm}^{-2}, 30 \mathrm{keV}\right)$ and laser annealed polycrystal Si sample. Regulated substrate temperature is $\mathrm{T}_{\mathrm{a}}=19^{\circ} \mathrm{C}$.

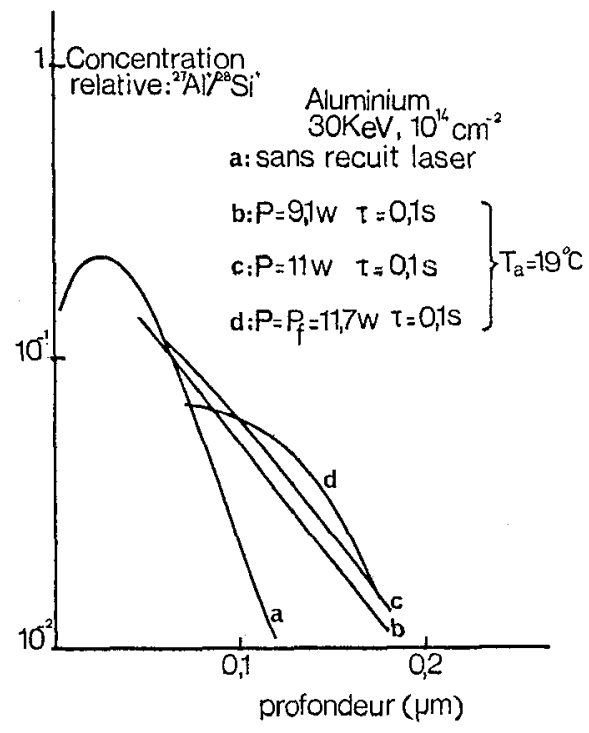




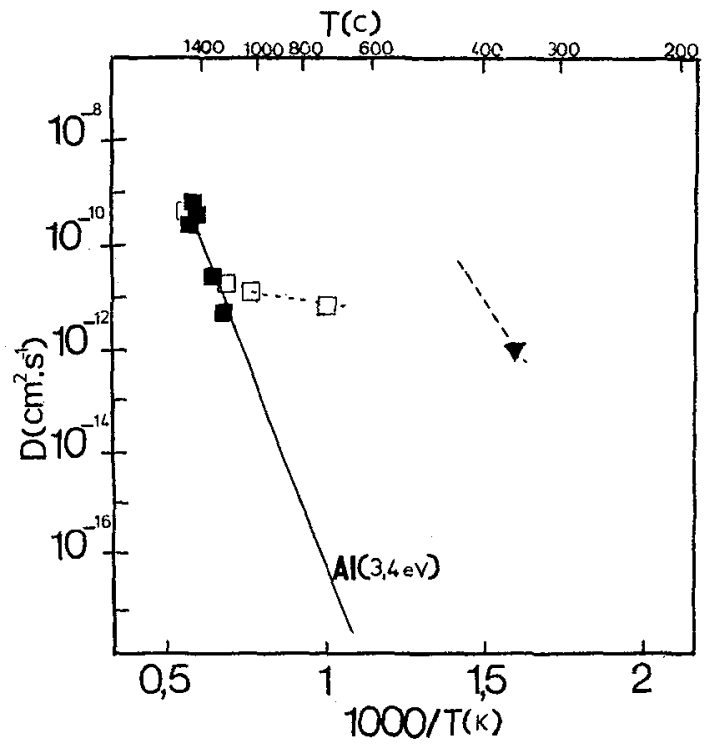

Fig. 4 - Coefficient de diffusion de l'aluminium dans le silicium en fonction de la température : Monocristal : réf. $/ 4,5 /, \square$ nos mesures. Polycristal $: \nabla / 10 /$, -.-- /6/; $\square$ nos mesures.

Temperature dependence of the diffusion coefficient of Al in $\mathrm{Si}:$ single crysta1 : ref. $/ 4,5 /$, our measurements. Polycrystal :

$\nabla / 10 /$ $16 /$

$\square$ our measurements.

La diffusion se manifeste par un abaissement et un décalage du profil SIMS d'une zone recuite, par rapport à une zone non recuite. Partant du profil avant recuit, une simulation sur calculateur nous conduit au profil après recuit pour différentes valeurs du paramètre $D t_{\text {eff }}$ : la comparaison du résultat de ce calcul avec I'expérience nous permet de déterminer $D t_{\text {eff. }}$. La température de 1 'expérience $\mathrm{T}_{\text {eff }}$ est connue, grâce au modèle thermique, par l'intermédiaire de la puissance laser $P$, et $t_{\text {eff }}$ est déduit de $\tau$ en utilisant également le modèle thermique $/ 3 /$. Nous en déduisons $\mathrm{D}\left(\mathrm{T}_{\text {eff }}\right)$, qui est reporté sur 1a Fig.4.

Les valeurs de $D(T)$ obtenues pour le monocristal $s^{\prime}$ alignent bien avec celles des auteurs antérieurs $14,5 /$. Ceci indique que les caractères particuliers du recuit laser (brièveté, gradients de température considérables, présence d'une certaine inhomogénéité latérale) ne modifient pas de façon importante le phénomène de diffusion, et que le modèle thermique utilisé en rend compte correctement.

Pour le polycristal, trois remarques sont à faire avant toute interprétation : - une forte concentration d'Al est trouvée en surface due à une exaltation du signal ${ }^{27} \mathrm{Al}^{+}$et à $\mathrm{la}$ diffusion de $\mathrm{Al}$ vers la surface. Nous $n^{\prime}$ utilisons donc pas cette partie du profil.

- la dose d'Al implanté étant plus faible, le signal aléatoire obtenu loin de la surface n'est plus négligeable, et a été soustrait du profil d'Al pour la présentation de la Fig. 3.

- I'énergie d'activation $\mathrm{E}_{a}$ étant inconnue a priori, et devant être introduite dans le modèle thermique, sa valeur réelle résulte d'une itération, qui conduit aux 
valeurs de $D(T)$ présentées sur 1a Fig. 4.

On voit que la diffusion intergranulaire est prépondérante en dessous de $1300^{\circ} \mathrm{C}$. Le coefficient de diffusion correspondant $D_{j}$ a une énergie d'activation $E_{j}$ que nous ne connaissons qu'avec imprécision, mais qui est en tout cas faible, inférieure à $1 \mathrm{eV}$. Physiquement $D_{j}$ résulte à la fois d'une diffusion intergranulaire proprement dite et d'une ségrégation de Al des grains vers les joints, que nous ne pouvons séparer. Notre courbe $D_{j}(T)$, extrapolée jusqu'à $350^{\circ} \mathrm{C}, n^{\prime}$ 'est pas en contradiction avec les valeurs $D_{j}\left(350^{\circ} \mathrm{C}\right)$ déterminées par NAKAMURA $/ 10 /$ et HWANG $/ 6 /$; qui sont de $1^{\prime}$ 'ordre de $10^{-12} \mathrm{~cm}^{2} / \mathrm{s}$. Par contre cé dernier trouve $\mathrm{E}_{j}=2,64 \mathrm{eV}$, valeur plus élevée que la nôtre, mais en même temps son terme préexponentiel $D_{j o}$ est $1,3 \cdot 10^{7} \mathrm{~cm}^{2} / \mathrm{s}$, valeur qu'il juge lui-même anormalement élevée.

En conclusion, dans la perspective de la "filière épitaxique" de photopiles, Al est une impureté dont le coefficient de diffusion à $1100^{\circ} \mathrm{C}$ reste inférieur à $10^{-11} \mathrm{~cm}^{2} / \mathrm{s}$,

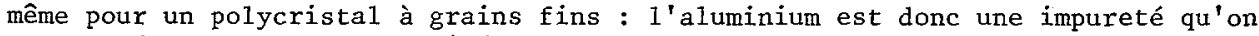
peut tolérer en grande quantité dans le substrat.

\section{REMERCIEMENTS}

Les auteurs remercient R. LEGROS, J. CHAUMONT, C. GRATTEPAIN et F. MAURICE pour avoir mis à leur disposition respectivement 1'appareillage de recuit laser, I'implanteur d'ions ainsi que les microanalyseurs ioniques de Bellevue et de Saclay.

\section{REFERENCES}

/ $1 /$ MATSumoto S., Gibbons J.F., Deline V. and EVANS C.A. Jr., App1. Phys. Lett. 37 (1980) 821.

/ $2 /$ STRUTHERS J.D., J. App1. Phys. 27(1956)1560.

/ 3 / LERAY C., BOUREE J.E., MOREAU R., RODOT M., J. Microsc. Spectrosc. Electron. 7 (1982) 235;

BOUREE J.E., LERAY C., RODOT M., in Laser-Solid interactions and transient

thermal processing of materials. Proceedings of M.R.S. Meeting, Nov.1982, Editors NARAYAN J., BROWN W.L. and LEMONS R.A.

/ 4 / FULLER C.S. and DITZENBERGER J.A., J. App1. Phys. 27 (1956)544.

/ 5/ SEEGER A. and CHIK K.P., Phys. Status Solidi B 29(1968) 455.

$/ 6 /$ HWANG J.C.M., HO P.S., LEWIS J.E. and CAMPBELL D.R., J. App1. Phys. 51(1980) 1576.

/ $7 /$ GOLD R.B. and GIBBONS J.F., J. App1. Phys. 51(1980) 1256.

/ 8/ LAX M., J. App1. Phys. 48(1977)3919;

LAX M., App1. Phys. Lett. 33(1978)786.

/ 9/ NISSIM Y.I., LIETOILA A., GOLD R.B. and GIBBONS J.F., J. App1. Phys. 51(1980) 274.

/10/ NAKAMURA K. and KAMOSHIDA M., J. Appl. Phys. 48(1977)5349. 